\title{
Crecimiento económico en términos de... ¿libertad o intervencionismo?: modelo nórdico versus modelo de los países del sur de Europa
}

\section{Economic growth in terms of ... freedom or interventionism?: nordic model versus model of the southern European countries}

\author{
Miguel Ángel García Gordillo* \\ FACULTAD DE ADMINISTRACIÓN Y NEGOCIOS, OBSERVATORIO DE POLÍTICAS PÚBLICAS, \\ UNIVERSIDAD AUTÓNOMA DE CHILE, SEDE TALCA
}

\section{RESUMEN}

El uso eficiente de recursos escasos debería ser un argumento suficiente para combatir al socialismo. Algunos gobernantes insisten en la figura del Estado como una condición necesaria en la preservación de los derechos de las clases más empobrecidas. Y en parte es cierto, ya que es muy difícil que una persona con escasos recursos pueda progresar cuando es el Estado quien criminaliza al progreso. Por otro lado, aquellos gobernantes que apuestan por liberalizar el mercado laboral y reducir el gasto público, consiguen que su política se refleje en un mayor progreso económico; con un mayor nivel y calidad de vida. En Iberoamérica, muchos países han buscado continuas fórmulas para tener un crecimiento sostenible. Chile ha sido un ejemplo de crecimiento económico para todos aquellos países que han imitado (en mayor o menor medida) un sistema pro-liberal, como ha sido el caso de su vecina Perú. No obstante, la actual clase política chilena piensa que ha llegado el momento de cambiar el sistema pro-liberal por un sistema más intervencionista, más europeo. Sin embargo, en ese recorrido ideológico se suele hacer referencia, únicamente, a los países nórdicos como aspiración política. Este trabajo pretende aclarar ciertos conceptos económicos y dar a conocer las implicaciones que serían necesarias si se quiere ser coherente con la aplicación de la política pública nórdica.

Palabras claves: Suecia, Estado, capitalismo, intervención, liberalismo.

\begin{abstract}
The efficient use of scarce resources should be a sufficient argument to combat socialism. Some rulers insist on the figure of the State as a necessary condition in the preservation of the rights of the poorest classes. And in part it is true, since it is very difficult for a person with limited resources to progress when it is the State that hinders such effort. On the other hand, those leaders who are committed to liberalizing the labor market and reducing public spending, tend to see their policy lead to greater economic progress; with a higher level and quality of life. In Latin America, many countries have tried to have sustainable growth during the last decades. Chile has been an example of economic growth for all those countries that have imitated (to a greater or lesser extent) a liberal system. However, the current Chilean political class thinks the time has come to change the liberal system for a more interventionist, more European approach. Although in this ideological journey, reference is usually made only to the Nordic countries as a political aspiration. This work aims to clarify certain economic concepts and to show the implications necessary to be consistent with the application of Nordic public policy.
\end{abstract}

Keywords: Sweden, State, capitalism, intervention, liberalism.

*miguelgarciagordillo@gmail.com 


\section{INTRODUCCIÓN}

uando se discute sobre si debería haber más o menos intervención del Gobierno en la economía... la gran olvidada es el mercado. En las últimas décadas se ha elevado el tono en cuanto a la necesidad de acercar a los más jóvenes hacia la idea de una progresiva educación financiera, y a su vez han surgido voces críticas sobre las consecuencias que estas medidas podrían repercutir en el conjunto de la sociedad. Dada la actual coyuntura económica global, advierto una falta de valores morales donde nuevas élites (mafias) aparecen para "pescar en río revuelto" en favor de sus propios intereses.

No es casual el uso de la palabra "interés", ya que viene a reflejar el lucro producido por el capital. Es decir, al hablar de interés, se hace mención a la capacidad que tiene el capital de generar más capital (Marx 2004, 99-104). Y en línea a esta breve introducción, tiene sentido resolver:

- ¿Qué es el capital?

- ¿Qué es el capitalismo?

- ¿Qué une o diferencia a ambos conceptos?

- ¿Está justificado el rechazo que ciertos economistas estatistas hacen del capitalismo?

La palabra capital proviene del latín "capitalis", que a su vez deriva del término "caput" (cabeza). Se trata, por tanto, de un concepto perteneciente o relativo a la cabeza. Así pues, dependiendo del ámbito al que hagamos referencia tendrá un sentido u otro, y con respecto al campo de la economía, es necesario distinguirlo del concepto de bienes de capital. Si bien, los bienes de capital hacen referencia a un bien concreto (tangible), el concepto de capital hace referencia a la estimación monetaria (intangible), a precios de mercado, de los factores o medios de producción (entre los que se encuentran los bienes de capital).

Es importante señalar que el dinero tal y como lo conocemos hoy día, no siempre existió, sin embargo, facilitó las relaciones comerciales como nunca se había visto. El dinero surge como alternativa al trueque (valor de cambio) ${ }^{1}$ en una etapa donde la 
mercantilización empezaba a resultar cada vez más compleja debido al creciente número de "commodities" que se acumulaban en los diferentes mercados para satisfacer las necesidades de sus propietarios, a través del libre intercambio. Así pues, establecer el valor de cambio resultaba cada vez más complejo debido a las infinitas posibles combinaciones de productos, y se terminó imponiendo la moneda como un nuevo producto capaz de reflejar el valor estimado ${ }^{2}$ de cada producto en base a la oferta y demanda generada para cada uno de los "commodities". Es así como, a través de un proceso de cooperación social y voluntario se inicia el efecto continuado de compra-venta de los factores de producción, mediante el uso del dinero (que ejerce como garante del valor ${ }^{3}$ de cada producto transado). De esta manera se produjo una transición comercial que dejaba atrás un sistema de mercantilización básico para pasar a un sistema basado en el capitalismo.

Atendiendo a las aclaraciones anteriores, se evidencia que el concepto de capitalismo no es más que un vocablo que describe, desde siempre, la manera en que los individuos del mundo establecen sus relaciones comerciales diarias. No obstante, este sistema capitalista no hubiera sido posible sin atender a la relevancia del concepto de capital y al uso del dinero. Cabe destacar que, aunque haya personas que asocian a Marx como el primer crítico mediático del capitalismo, lo cierto es que ponía el foco de sus críticas en el capital. Así pues, es necesario aclarar que la persona que popularizó el concepto de capitalismo fue el economista alemán Werner Sombart, cuando en 1902 escribió su obra maestra "Der moderme Kapitalismus" (El capitalismo moderno). En este sentido, definió al capitalismo como un sistema económico determinado, que se podía caracterizar de la siguiente manera: "es una organización económico-comercial, en la cual colaboran regularmente dos grupos distintos de la población, los poseedores de los medios de producción, que al mismo tiempo tienen la dirección y que son los sujetos económicos, dominados por los principios del lucro y del racionalismo económico y los proletarios, que solo trabajan, como objetos económicos”. También mencionaba que ambos elementos, estaban ligados por el contrato 
del trabajo, que es regulado por la situación eventual del mercado del trabajo. En este punto cabía añadir que cada día eran más las personas que se desarrollaban profesionalmente como "proletarios" y a la vez como "poseedores de medios de producción".

Si bien es cierto la necesidad de corregir a Werner Sombart en su idea de ver al capitalismo como un proceso de maduración o evolutivo, también se antoja necesario desmontar otros relatos falaces y nocivos. A lo largo del último siglo se han difundido cuantiosas críticas sobre el sistema capitalista por parte de numerosos economistas estatistas, destacando la falta de fundamento y el generoso elenco de falacias que contienen sus argumentos. Es habitual ver cómo al intentar fundamentar las críticas hacia dicho sistema, se revela verdaderamente una crítica sobre la moral de los consumidores, la ética profesional o el poder ejercido por las instituciones públicas que corrompen el libre mercado, lo cual, no tiene nada que ver con el capitalismo. Sin embargo, no resulta mediático para los medios de comunicación señalar al consumidor como responsable de sus pocos o muchos aciertos en el mercado y sí, acusar a aquellos que generan valor y que han conseguido triunfar en este gran circo romano.

A continuación, se ejemplifica a través de diversos autores cómo se "retuerce" el concepto de capitalismo para dotarlo de un tinte peyorativo:

- El político e historiador argentino Eduardo Sartelli describe al capitalismo como un sistema que nació y se ha mantenido siempre sobre la base de la violencia extrema.

- Por otro lado, el economista espańol y gran defensor de las teorías marxistas, Xavier Arrizabalo, concentra sus críticas hacia el capitalismo tomando como ejes centrales de su discurso; la ética profesional, la legislación empresarial, la carga impositiva, la desigualdad social, la explotación del proletariado, etc.

- Thomas Piketty, economista francés y reconocido mundialmente por su libro "El capital del siglo XXI" (Piketty 2014, 448) revela que la crisis financiera que explotó en 2008 es la mayor crisis del capitalismo desde la crisis de 1929. 
- Otras tantas falacias como aquellas que asocian al capitalismo con la idea del "juego de suma cero", donde se afirma que la acumulación de la riqueza siempre beneficia a los mismos a la vez que provoca una mayor vulnerabilidad entre los pobres. Otras falacias como las que asocian al consumismo con capitalismo.

- También, el reconocido y prestigioso economista indio, Amartya Sen reconoció, erróneamente, la inexistencia del sistema capitalista (como sistema íntegro de cualquier sistema económico). Para ello se apoyó en la falacia ad antiquitatem. Participando en la Conferencia Internacional sobre Desarrollo Humano y Enfoque de Capacidades, titulada "Desarrollo Humano: vulnerabilidad, inclusión y bienestar”, en Managua, 2013, declaró:

"Algunos alegan que se deben nacionalizar todos los medios de producción, entonces no hay ningún pais socialista en el mundo. Otros dicen que el capitalismo significa que todos los mercados deben ser privados, pero tampoco hay un país asi”.

Aunque todos los autores anteriores podrían considerarse dentro del espectro estatista, existe otro eje político -liberalismo(Lomasky 1987, 31-34) donde, sin perseguir los mismos fines de desprestigiar su significado, sus mayores representantes también terminan dotando al capitalismo de elementos impropios. El economista estadounidense Murray Rothbard popularizó el término anarcocapitalismo como una filosofía que aúna la propiedad privada y el libre mercado como las piedras angulares .

Dejando de lado a los libertarios, ninguno de los planteamientos anteriores tiene cabida en la crítica al capitalismo, ya que el capitalismo no es resultado de una acción que tiene el propósito de mejorar el bienestar de la sociedad, sino que consiste en un sistema eficaz que facilita el intercambio voluntario de infinitos "commodities", tal y como se mencionó anteriormente. Y a tenor de los resultados, se evidencia que no hay hasta la fecha un sistema alternativo que provea progreso en los mismos términos. 
En tanto las sociedades han evidenciado un crecimiento económico, la mayoría de estos autores estatistas hacen mención a una mayor participación de los diferentes gobiernos como agentes garantes de "derechos sociales" 4 . Si bien no deja de ser una falacia y casi un oxímoron atendiendo al concepto de Estado por parte de Max Weber (define Estado como un conjunto de instituciones públicas que reclaman para sí, el monopolio de la violencia física legítima), sí es preciso reconocer la gran cantidad de países desarrollados y en vías de desarrollo que, hoy en día, cuentan con un Estado superlativo. Y todo ello tiene un origen y un contexto histórico. En el último siglo, tras el crack de 1929 en Estados Unidos, surgió una corriente pro-intervencionista liderada por el matemático (no economista) J.M. Keynes quien exploraba una alternativa viable a través de la gestión y supervisión política con el propósito de suavizar los efectos de los recurrentes ciclos económicos. La aplicación de sus teorías promovía la necesidad de un Estado omnipresente y así, el propio Keynes reconocía en el prefacio de su libro "La teoría general de la ocupación, el interés y el dinero" (año 1936, edición en alemán), lo siguiente: "No obstante, la teoría del producto en su totalidad que este libro tratará de ofrecer es, por mucho, más fácilmente adaptable a las condiciones de un Estado totalitario que a la teoría de la producción y distribución de un producto dado bajo las condiciones de libre competencia y en buena medida de Laissez-Faire".

Entre sus teorías trataba de explicar los efectos de la recesión económica aludiendo a la falsa paradoja del ahorro 5 , y justificaba con ello la estimulación prolongada del consumo obviando la libertad de decisión de los agentes económicos, los cuales deberían ser obligados a consumir para mantener la demanda y por consiguiente, evitar la pérdida de empleo. Para aflorar el ahorro, Keynes se apoya en la subida de impuestos, la política inflacionista, combinación de deuda y gasto públicos (destinado al consumo, inversión y obra pública, principalmente). Sin embargo, al no considerar la importancia de la variable del tiempo, la aplicación las teorías keynesianas perjudica a las etapas productivas más alejadas del consumo, las cuales necesitan nutrirse del ahorro presente para una producción futura sostenible. 
Las consecuencias derivadas de castigar el ahorro se reflejan en aquellas economías que han seguido fielmente sus teorías; como es el caso de la actual Argentina, con multitud de servicios públicos, inflación acumulada del $36 \%$ en el presente año, déficit público estructural, tasa de desempleo en torno al $12 \%$ y deuda calificada como "default". En definitiva, se incrementan o aparecen nuevos colectivos vulnerables que quedan al margen del mercado y por lo tanto terminan siendo dependientes del Estado para "subsistir", lo que complica poder salir de esa dinámica.

Además, basta una simple visión macroeconómica y comparativa para mostrar cómo la vía más rápida para mejorar el bienestar de cada individuo (y por lo tanto el del conjunto de la sociedad) implica, como máxima prioridad, una mayor liberación política (menor peso de los gobiernos en las decisiones diarias que afectan a los ciudadanos). Algunos de los requisitos en el corto y medio plazo para crecer en lo económico y en lo social pasan por:

1) un mercado libre (libertad individual), lo que implicaría eliminar tanto la asimetría de información como las regulaciones estatales que suponen barreras de entrada para la libre competencia,

2) garantía por la preservación del derecho a la legítima propiedad privada,

3) ahorro, sea generado de manera interna o externa y dedicarlo a la inversión,

4) un entorno de certidumbre y seguridad contractual y,

5) una menor carga burocrática para llevar a cabo iniciativas privadas de emprendimiento.

Y en el largo plazo se podría mencionar como esencial la necesidad de alcanzar un equilibrio presupuestario, evitando así situaciones de quiebra técnica y por lo tanto, evitar la mala imagen proyectada sobre potenciales inversores en el territorio nacional.

En este punto, se puede arrojar información extraída de la "Fundación Heritage" (EFI 2020) (recolección de datos desde junio del 2018 a junio del 2019), quien se encarga de recopilar datos nacionales de decenas de países participantes y que son clasificados en 12 dimensiones, como son: derecho de propiedad, 
efectividad judicial, integridad gubernamental, carga fiscal, gasto gubernamental, salud fiscal, libertad empresarial, libertad laboral, libertad monetaria, libertad comercial, libertad de inversión y libertad financiera. Adicionalmente existe un índice global que permite comparar a los distintos países analizados en torno a la libertad económica de cada uno, (índice de libertad económica) donde los valores pueden oscilar entre 0 y 100 (más cercano a 100 corresponde con países con mayor libertad económica). En la siguiente tabla se muestran los seis países con mayor libertad económica (se añade la posición de Chile y la del país con menor libertad económica entre los analizados) según el último ranking elaborado (Ver tabla 1).

Tabla 1: PAíses SEgún El ÍNDICE LiberTad ECONÓMiCA (MEdiAdos 2OI 8 - MEDIADOS 20I9).

\begin{tabular}{|c|c|c|c|}
\hline Posición mundial & Posición regional & País & Índice libertad económica $\infty$ \\
\hline 1 & $1^{\dagger}$ & Singapur & 89,4 \\
\hline 2 & $2^{\dagger}$ & Hong Kong & 89,1 \\
\hline 3 & $3^{\dagger}$ & Nueva Zelanda & 84,1 \\
\hline 4 & $4^{\dagger}$ & Australia & 82,6 \\
\hline 5 & $1^{¥}$ & Suiza & 82,0 \\
\hline 6 & $2^{¥}$ & Irlanda & 80,9 \\
\hline$\ldots$ & $\ldots$ & $\ldots$ & $\ldots$ \\
\hline 15 & $2^{\mathrm{r}}$ & Chile & 76,8 \\
\hline$\ldots$ & $\ldots$ & $\ldots$ & $\ldots$ \\
\hline 180 & $42^{\dagger}$ & Corea del Norte & 4,20 \\
\hline
\end{tabular}

* Irak, Libia, Liechtenstein, Somalia, Siria y Yemen no fueron analizados.

$\dagger$ Región: Asia y pacífico.

$¥$ Región: Europa.

r Región: Continente americano. Chile mejora 3 puestos a nivel mundial, pasando de 75,4 (18)

a 76,8 (15) en un solo ańo.

$\infty$ Rango de puntuaciones de libertad económica:

$0-49,9$; reprimido.

50-59,9; mayoritariamente reprimido.

60-69,9; moderadamente libre.

70-79,9; mayoritariamente libre.

80-100; libre.

Algunas críticas mencionan el sesgo ideológico que pueda haber en torno a los criterios seleccionados y la metodología empleada en sus análisis. Sin embargo, la réplica es tan sencilla como 
incontestable: «Realicen sus propios estudios y compartan sus resultados». No obstante, no son pocos los organismos públicos y privados que publican resultados macroeconómicos y estos, pueden utilizarse para ver cómo se relacionan estos datos con relación a la posición de libertad económica que tienen los respectivos países. En este sentido, corresponde ver si existe alguna relación entre la libertad económica y otros indicadores que midan aspectos de satisfacción, progreso o desigualdad. Así pues, el Banco Mundial ofrece como datos más actualizados los del año 2018. En cuanto al análisis de la desigualdad de ingresos o gastos dedicados al consumo, el índice de Gini (un mayor valor en el índice reporta una mayor desigualdad en términos de ingresos) releva que sólo Singapur (de los países con mayor libertad económica) está en el puesto 36/103 (con una puntuación de 39,8 siendo la máxima de 57,7 para Sudáfrica y la mínima de 24,4 para Islandia) con mayor desigualdad de ingresos. Una explicación podría ser debido a que se trata de un país con una economía emergente. Por otro lado, Chile se encuentra en el puesto número 13/108 (con una puntuación de 45,9), lo que invita a una reflexión sobre cómo abordar el mercado laboral. El país ha mejorado muchos indicadores en las últimas décadas, pero debe enfrentar la subida salarial mediante un incremento de la competencia y la productividad y no, mediante regulaciones estatales. Dos vías para mejorar ambas variables pasan por adoptar una cultura laboral diferente y a través de un aumento de inversión en bienes de capital en etapas anteriores a la del consumo final. Por otro lado, países como Nueva Zelanda, Australia, Suiza e Irlanda se sitúan entre las posiciones con menor desigualdad de ingresos, como cabría esperar. Finalmente, Hong Kong y Corea del Norte no reportaron valores para el índice de Gini (IDI 2018).

Otro indicador que se puede analizar y que es muy demandado por los sectores más populistas es el índice de "progreso social" (año 2018, para reportar datos atendiendo al mismo año que los reportados para el índice de Gini, que son los datos más actualizados disponibles) que recoge dimensiones de desempeño social y ambiental (los valores más altos -primeros puestos- corresponden 
con un mayor nivel de progreso). En este listado tenemos, excepto a Hong Kong (no reportó valores), a los primeros países en libertad económica entre los primeros 29 puestos de un total de 163 países analizados (posición en términos de progreso social: $5^{\circ}$ Nueva Zelanda, 9o Suiza, $7^{\circ}$ Australia, $11^{\circ}$ Irlanda y 29 Singapur). Luego, se encuentra Chile en el puesto $31^{\circ} \mathrm{y}$ por último, los valores para Corea del Norte lo sitúan en el puesto $141^{\circ}$ (SPI 2018).

De acuerdo con los índices de libertad económica, índice de Gini e índice de "progreso social”, cabría esperar una sociedad más responsable, menos ignorante respeto al conocimiento político y sus implicaciones, con mayores ansias de libertad... y todo ello para poder empezar a reflexionar sobre el verdadero rol del Gobierno como garante de "derechos y libertades". Éste nos impone a través de impuestos, tasas y contribuciones varias, entregar una parte de nuestro trabajo que irá destinada al mantenimiento de las estructuras de poder, lo cual recuerda bastante a la errónea teoría de explotación (teoría del valor trabajo) que mencionaba Marx en su crítica al "capitalismo". La actualidad chilena tiene mucho que decir en este sentido, debido principalmente al plebiscito celebrado en el 2020 que dio inicio a un proceso constituyente para redactar una nueva Constitución. Chile cuenta hoy más que nunca, con personas formadas y experimentadas en un extenso elenco de especialidades, pero habrá que ver la capacidad que tienen para consensuar un sistema político que otorgue un crecimiento económico sostenible en el largo plazo. En la última década, el panorama político nacional parece haber dado un giro a la izquierda y como consecuencia de ello, anteponer el relato populista a los datos podría suponer una nueva etapa de mayor deuda pública, consolidación de los estamentos más empobrecidos de la sociedad, mayores niveles de criminalización, pérdida de identidad de la sociedad chilena... y un largo etcétera, Por ello, es importante evitar problemas que ya están arraigados en otras economías del mundo. 


\section{Desarrollo}

Existen ciertos paradigmas que han sido introducidos en el relato popular y que desde su inicio fueron utilizados para atender a las conveniencias de las élites políticas (la conveniencia de tener un Estado cada vez mayor y con más capacidad coercitiva). Una gran cantidad de esos paradigmas están relacionados de manera directa o indirecta con los indicadores macroeconómicos que se han citado anteriormente. Algunos de estos paradigmas están orientados a pensar que los ricos siempre son ricos, que el esfuerzo siempre debe verse recompensado (teoría del valor trabajo), que los países nórdicos son socialistas, que pagar impuestos es vital para mantener la educación, que la pobreza se podría solucionar imprimiendo dinero, que para trabajar en una empresa es imprescindible tener un título formativo, que el producto interior bruto debe centrarse en el valor ańadido para evitar la doble contabilidad, etc. Sin embargo, es necesario poder cuestionar todas y cada una de estas creencias.

El caso de Suecia es recurrente ya que ha sufrido ciclos económicos a lo largo de los últimos 150 ańos y que ponen en tela de juicio algunos de estos paradigmas, como aquel que señala que Suecia llegó a ser rico gracias a una gestión política socialista ${ }^{6}$. Sin embargo, la realidad es otra, Suecia es un país que ha evidenciado un mayor progreso en periodos de una mayor libertad económica; al igual que sucedió en Chile o recientemente en Irlanda, Singapur, etc. Pero también ha demostrado cómo se puede estancar su economía en épocas de mayor intervencionismo, con la excusa del mal llamado "Estado del Bienestar". Cuando el proceso de intervención no tiene límites, los resultados no se hacen esperar, tal y como sucedió en la Cuba castrista y la Venezuela chavista o, como está sucediendo en la Argentina iniciada en la etapa peronista o, en la Espańa sanchista, es decir, desde que Pedro Sánchez llegara a la Presidencia del Gobierno en enero del 2020.

Partiendo de un origen humilde a inicios del siglo XIX, Suecia empezó su crecimiento en términos agregados, aunando esfuerzo, sacrificio, capacidad de ahorro, planificación... a nivel individual. Así, en 1950, Suecia contó con una subida notable de ingresos per 
cápita sobre el PIB, llegando en la década de 1970 a estar considerado como uno de los países más ricos respecto al resto del mundo. A partir de 1980, el Estado se organizó para aumentar su tamaño y su capacidad de interferir en las decisiones de los demás agentes económicos.

\section{Gasto público y tasa de crecimiento real (\% de PIB).}

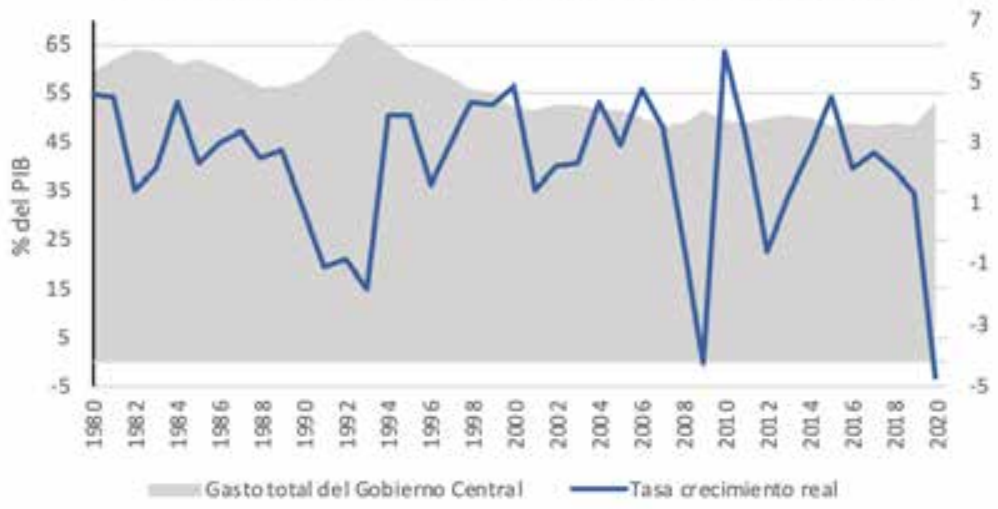

Figura 1. Suecia - Gasto total del Gobierno Central y tasa de crecimiento real sobre el porcentaje del Producto Interior Bruto.

Tal y como se aprecia en la figura 1, Suecia (en líneas generales) ha disminuido su gasto público sobre el PIB en un 20\% desde 1993. No obstante, cada vez que Suecia optó por incrementar el tamaño de las instituciones públicas, derivó en una recesión económica caracterizada básicamente por un efecto negativo sobre la tasa de crecimiento real, y lo hizo de manera muy elástica en proporción a las alteraciones del gasto público. En concreto, vemos que durante el periodo que va entre 1990 y 1992, se da el mayor crecimiento de gasto público en términos relativos (llegando a representar el 66,3\% del PIB en 1992), implicando una reducción de la tasa de crecimiento real con un impulso 17 veces mayor (con una tasa de crecimiento negativa de $0,9 \%$ al final del mismo periodo). De manera similar suceden otros periodos en los que el Estado aumentó el gasto público, como fue en 1980-1982, 2008-20097 y 2019-2020 (OECD 2021). 
En la siguiente figura (Figura 2) se ha realizado una comparativa con Grecia ${ }^{8}$. Ambos países están bajo el paraguas de la Unión Europea (con todo lo que eso supone), también reportan un censo de población en torno a 10,5 millones de habitantes, recaudan en conceptos de ingresos fiscales un porcentaje similar $(27,91 \%$ vs 25,54\% del PIB para Suecia y Grecia respectivamente) y durante los últimos 20 años ha mantenido un parejo nivel de gasto público sobre el total del PIB (Grecia sufrió un incremento del 20\% - 25\% del gasto público desde 1990 al 2000). Visto así, también se podría decir que Grecia es igual de "socialista" que Suecia y por lo tanto, sus ingresos reales per cápita deberían seguir una tendencia similar. Sin embargo, la tasa de crecimiento real de Grecia sufre desde 2009 hasta la actualidad una recesión más acentuada y prolongada en el tiempo. Es decir, los ingresos reales per cápita para Suecia y Grecia en 2019 (a precios constantes del 2010) fueron de 59.461 dólares y 23.620 dólares, respectivamente (OECD 2021).

\section{Comparativa entre Suecia y Grecia, por variables de gasto} público y tasa de crecimiento real (\% del PIB).

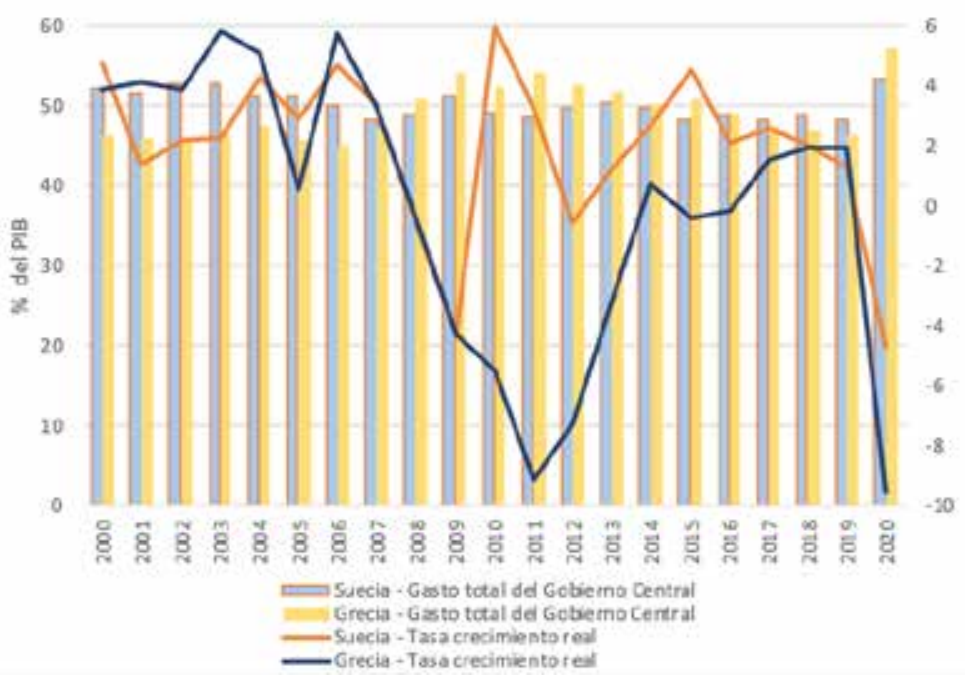

Figura 2. Comparativa entre Suecia y Grecia, por variables de gasto público y tasa de crecimiento real (\% del PIB), para el periodo 2000-2020. 
Entonces, ¿a qué podría deberse tal diferencia en países que en principio son comparables? Es importante entender que la ciencia económica sigue una metodología apriorística9 deductiva, y por lo tanto, está lejos de ser una ciencia empírica. No obstante, la economía positiva permite explorar algunas teorías en la interpretación de los hechos.

Así pues, se suele argumentar que los países nórdicos, como sería el caso de Suecia, recaudan la mayor parte de sus ingresos tributarios a partir de las clases más acaudaladas, con impuestos progresivos. No obstante, no es exactamente así. La estructura impositiva de un país consiste en dividir la recaudación en función del concepto que se grava. De esa manera, la recaudación proviene de impuestos al consumo (principalmente IVA e impuestos especiales), impuestos sobre las rentas del trabajo (básicamente IRPF -ISR en Hispanoamérica- y cotizaciones sociales) e impuestos sobre el capital (lo que vienen siendo rentas del capital e impuestos sobre el patrimonio). Se tiene una idea equivocada al pensar que las personas que más riqueza acumulan son las mismas que mantienen o deben mantener el "Estado del Bienestar". En la siguiente tabla (Tabla 2) se expresan los porcentajes de impuestos que una persona paga en relación con el Producto Interior Bruto (Taxation and Customs Union, 2020).

Tabla 2. Origen de los ingresos tributarios, \% Del PIB.

\begin{tabular}{|c|c|c|c|c|}
\hline Grecia & \multicolumn{2}{|c|}{2005} & \multicolumn{2}{|c|}{2018} \\
\hline Impuesto al consumo & $11 \%$ & \multirow{3}{*}{ Total $31.9 \%$} & $14.6 \%$ & \multirow{3}{*}{ Total $38.9 \%$} \\
\hline Impuesto al trabajo & $13.5 \%$ & & $16.1 \%$ & \\
\hline Impuesto al capital & $7.4 \%$ & & $8.2 \%$ & \\
\hline Suecia & \multicolumn{2}{|c|}{2005} & \multicolumn{2}{|c|}{2018} \\
\hline Impuesto al consumo & $12.1 \%$ & \multirow{3}{*}{ Total $46.6 \%$} & $12.2 \%$ & \multirow{3}{*}{ Total $43.8 \%$} \\
\hline Impuesto al trabajo & $27.7 \%$ & & $25.5 \%$ & \\
\hline Impuesto al capital & $6.8 \%$ & & $6.1 \%$ & \\
\hline
\end{tabular}

La tabla refleja dos periodos (2005 y 2018) ya que Grecia tuvo que ser rescatada por su pésima gestión política. Por lo tanto, es preciso ver la realidad de Grecia antes de ser intervenida 
económica y financieramente y también cómo se ha reestructurado impositivamente tras un rescate que ha durado 8 ańos (2010-2018). Como se puede apreciar, en 2005 la diferencia en la recaudación era de $14,7 \%$ del PIB y tras la reestructuración impositiva, se redujo al 4,9\% del PIB. Sin embargo, el origen de los ingresos tributarios muestra las siguientes características:

- Los impuestos a las rentas del trabajo son las que más aportan respecto al $\mathrm{PIB}$, seguido de los impuestos al consumo y finalmente del capital.

- Los impuestos al consumo son los impuestos que menos discriminan en el conjunto de la sociedad y sin embargo, son el segundo concepto que más recauda para el Estado (En Grecia se ha incrementado notablemente en los últimos lustros: en 2004, el IVA tenía un tipo general del 18\% y un tipo reducido del $8-4 \%$ y, actualmente tiene el general en $24 \%$ y tipo reducido del 13-6\%. Por otro lado, Suecia lleva desde 1996 con un tipo general del $25 \%$ y tipo reducido del $12-6 \%)$.

- Los impuestos al capital son los impuestos que más discriminan en el conjunto de la sociedad y sin embargo, son el concepto que menos recauda para el Estado.

- Los impuestos al trabajo ${ }^{10}$ son aquellos que más recaudan en el conjunto de la sociedad, sin embargo, en este punto se abren dos hipótesis:

- $\quad$ Los que pagan más impuestos son aquellos que más ingresos reportan (Impuestos progresivos efectivos).

- La sociedad está compuesta básicamente por una clase media-alta y por lo tanto, la recaudación de este impuesto siempre será alta ya que va en función de los ingresos generados.

De acuerdo con este punto sobre los impuestos a las rentas del trabajo, es relevante aclarar que las diferentes clases sociales (clase baja, media y alta) son clasificadas en función de los ingresos. Así, la Organización para la Cooperación y el Desarrollo Económico (OCDE), clasifica las clases medias como aquellos hogares unifamiliares que ingresen entre el $75 \%$ y el 200\% de la renta 
mediana de la población nacional (OECD, 2019). Por lo tanto, las clases menos pudientes serían aquellos que ingresen por debajo del $75 \%$ de la renta mediana y las clases más acaudaladas serían aquellas que tengan unos ingresos superiores al 200\% de dicha renta. En el informe presentado por la OCDE, se recogen datos del grado de participación en los impuestos sobre las rentas del trabajo (año 2015 para los casos de Suecia y Grecia), Con respecto a Suecia, la clase media proporciona el 69,1\% de todos los impuestos directos que pagan los hogares unipersonales en rentas del trabajo (Excluyendo las cotizaciones sociales a cargo del empleador), las clases más bajas aportan el 9,3\% y las más pudientes el 21,5\%. Al comparar los datos de Suecia con Grecia, se aprecia un reparto similar entre las distintas clases sociales (las clases bajan aportan el 14,1\%, las clases medias aportan el 60,25 y las clases más acaudaladas el 25,7\%), de hecho, esa similitud es consecuencia de las condiciones que fueron exigidas por el Eurogrupo para ser rescatada económica y financieramente del 2010 al 2018. En la siguiente figura (Figura 3) se muestra el reparto de la recaudación en los impuestos de la renta según un listado de países, distinguiendo; por un lado, los países clásicos del sur de Europa (Portugal, Espańa, Italia y Grecia) y por otro, los países nórdicos (Suecia, Dinamarca, Noruega, Finlandia e Islandia).

\section{Participación de las clases sociales en la recaudación del impuesto sobre las rentas del trabajo}

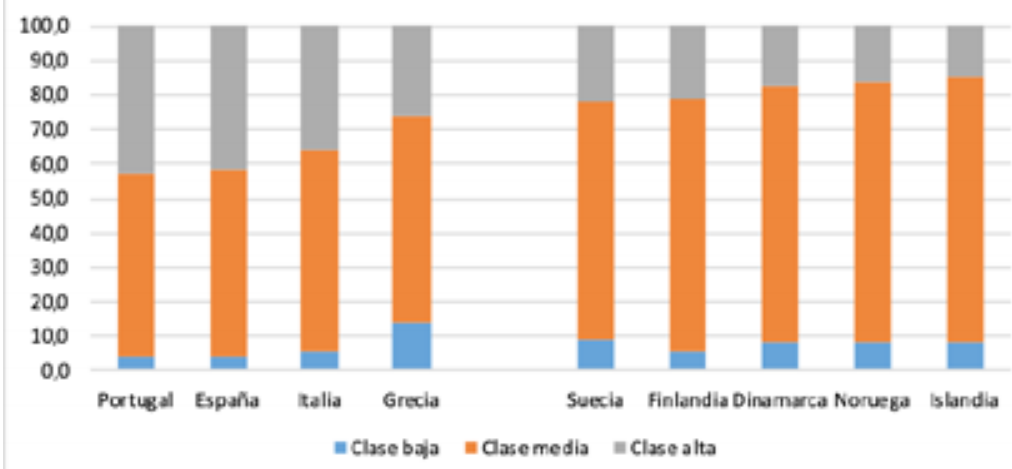

Figura 3. Reparto según las clases sociales (baja, media y alta) de la recaudación por el impuesto sobre rentas del trabajo. Gráfico adaptado del informe de la OCDE: "Under 
Pressure: The Squeezed Middle Class", en 2019. Datos recogidos en 2014 para Islandia, Italia y Noruega y, 2015 para Portugal, España, Grecia, Suecia, Finlandia y Dinamarca).

Atendiendo a los promedios de ambos grupos, en la medida que los países del sur quisieran parecerse a los países nórdicos, deberían recaudar un punto más de las clases más baja, deberían hacer lo mismo con las clases medias un $17,2 \%$ y por último deberían reducir la recaudación por parte de las clases más acaudaladas en 18,3\%. El informe de la OCDE también permite saber cuál sería el nivel de aporte de la clase media griega en comparación con Suecia, manteniendo el peso actual de su clase media, ya que la clase media sueca es significativamente mayor a la griega $(70,9 \%$ vs. $62,4 \%$ respectivamente). En ese caso, la clase media griega debería aportar un $0,6 \%$ más de lo que aportan actualmente. De aplicar la misma comparación entre los países del sur y los nórdicos, las clases medias de los países del sur deberían incrementar sus aportaciones en un $4,4 \%$.

Otro punto relevante en cuanto al rol que debe cumplir el Estado está relacionado con el empleo. Cuando un Gobierno centra sus esfuerzos en generar empleo de manera directa (a través de la creación de empleo público, expropiación de factores de producción, etc.), en el largo plazo termina siendo perjudicial para el conjunto de la sociedad. La inmensa mayoría de países cuentan con servicios prestados por instituciones públicas que no están estrictamente regidas por la ley de demanda y oferta, lo que deriva en el mantenimiento, a largo plazo, de puestos de trabajo de poco o nulo valor añadido. Por esa razón, el Estado debería centrarse en aplicar una política que favorezca la iniciativa privada, ya sea a través de una mayor digitalización del país, bajos impuestos, mejorar la imagen del país con buenos representantes, estabilidad política, etc.

Por otro lado, aunque exista una multitud de opciones para afrontar los retos macroeconómicos, los países nórdicos tienen una máxima para mantener el denominado "Estado del Bienestar"; «si se adopta una política de fronteras abiertas, no puede haber Estado del Bienestar; y si hay Estado del Bienestar, debe haber un 
requerimiento riguroso (ć́vico y legal) para autorizar la entrada y permanencia al país por parte de cualquier extranjero». Esta máxima permite a los países tener un mayor control del gasto y no caer en la insostenibilidad de la deuda pública. Así es como su tasa de deuda pública ha seguido una línea descendente desde 1995 y, para el 2020 su tasa de deuda pública fue del $41.9 \%$ del PIB. En cambio, Grecia ha seguido una línea ascendente que ha colocado su deuda en el 205.2\% del PIB (sin olvidar que durante este periodo de tiempo ha sido rescata hasta en tres ocasiones por el Eurogrupo). De hecho, los países del Mediterráneo (España, Italia y Grecia, principalmente) sufren las consecuencias de su errática gestión del gasto público cuando tratan de financiarse a través de los mercados, con altas tasas de interés exigidas por el resto de los agentes económicos (con un rendimiento del bono a 10 años mayor para Grecia comparado con Suecia, $1 \%$ vs $0,4 \%)$.

Este trabajo ha tratado poner de relieve algunas aclaraciones conceptuales y poner de manifiesto que cuando algunos partidos, concretamente en Iberoamérica, abogan por el "socialismo de los países nórdicos", en verdad el socialismo que defienden y que tratan de imponer es el de los países del sur de Europa; como Grecia, un país rescatado económica y financieramente durante casi una década (2010-2018) por haber llevado a cabo una política de incremento del gasto público (mayor "Estado del bienestar"), "criminalización" del libre mercado, fronteras abiertas a los inmigrantes ilegales, altos impuestos a las clases más acaudaladas, etc. La siguiente tabla (Tabla 3) resume las diferencias entre Suecia y Grecia, es decir, refleja las diferencias entre una economía sostenible y "liberal" frente a una economía rescatada, dependiente, socialista e..."intervenida”.

Tabla 3. Cuadro comparativo entre Suecia y Grecia con RESPECTO A ALGUNOS INDICADORES MACROECONÓMICOS.

\begin{tabular}{|l|c|c|}
\hline \multicolumn{1}{|c|}{ Indicador (ańo) / País } & Suecia & Grecia \\
\hline PIB per cápita US\$ $(2019)^{\beta}$ & $51.404 \$$ (puesto 13/190) & $19.570 \$$ (puesto 43/190) \\
\hline Índice de Gini $(2018)^{\infty}$ & $25.7 \%$ (puesto 102/107) & $33.2 \%$ (puesto 74/107) \\
\hline Facilidad iniciar un negocio $(2019)^{\alpha}$ & 82.02 (puesto 10/188) & 67.41 (puesto 77/188) \\
\hline Tasa desempleo $(2019)^{\pi}$ & $6.8 \%$ (puesto 41/103) & $17.3 \%$ (puesto 5/103) \\
\hline
\end{tabular}




\begin{tabular}{|l|c|c|}
\hline \multicolumn{1}{|c|}{ Indicador (ańo) / País } & Suecia & Grecia \\
\hline Tasa de pobreza $(2017)^{\mu}$ & $0.2 \%$ (puesto 47/66) & $0.9 \%$ (puesto 31/66) \\
\hline Índice libertad económica $(2019)^{¥}$ & 74.9 (puesto 22/180) & 59.9 (puesto 100/180) \\
\hline Índice competitividad global $(2019)^{\sim}$ & 81.25 (puesto 8/141) & 62.58 (puesto 59/141) \\
\hline
\end{tabular}

B El Producto Interno Bruto está en precios corrientes. Se tiende a señalar que cuanto mayor es el PIB per cápita, más rico es un país (En los puestos, mientras más cercano al primer puesto, mejor).

$\infty$ Mientras más bajo sea el porcentaje, menor desigualdad en la distribución del ingreso (En los puestos, mientras más cercano al primero puesto, peor).

$\alpha$ Un valor más cercano a 100 , indica una mayor facilidad para iniciar un negocio, y un valor cercano a 0 , supone mayores dificultades a la hora de iniciar un negocio (En los puestos, mientras más cercano al primer puesto, mejor).

$\Pi$ Un porcentaje elevado implica un mayor número de personas que estando en condiciones de trabajar y estando en búsqueda de trabajo, no consiguen hacerlo (En los puestos, mientras más cercano al primer puesto, peor).

$\mu$ Indica la proporción de personas que viven con menos de 1.9 dólares norteamericanos al día. Un mayor porcentaje implica una mayor cantidad de personas en la pobreza extrema (En los puestos, mientras más cercano al primer puesto, peor).

¥ Mientras mayor sea el puntaje, mayor libertad económica del país (En los puestos, mientras más cercano al primer puesto, mejor).

$\approx$ Un mayor puntaje, indica un mejor aprovechamiento de los recursos de un país (En los puestos, mientras más cercano al primer puesto, mejor).

\section{Conclusión}

En conclusión, los países que tratan de progresar económicamente no deberían dudar si hacerlo mediante un mayor grado de libertad o si hacerlo a través de más intervencionismo. Aquellos países con mayor libertad económica consiguen tener un mayor crecimiento del bienestar individual -en términos per cápita- (y por consiguiente, bienestar en términos agregados). Por otro lado, entre las personas que sufren de un mayor grado de intervencionismo estatal, se genera una sensación de estar pagando un cheque en blanco en favor de una vida en la que apenas pueden decidir por las cosas que le afectan individualmente, como por ejemplo ¿por qué se sigue aceptando que el Estado gestionará mejor el dinero (gran parte) que ellos ganan legalmente con sus trabajos? 
4 Referencias bibliográficas

EFI 2020. The economic freedom index. https:/www.heritage. org/index/pdf/2020/book/2020_IndexofEconomicFreedom_ Highlights.pdf (pag. 2) (Visto última vez 19-11-2020).

IDI 2018. The inclusive development index.. http://www3. weforum.org/docs/WEF_Forum_IncGrwth_2018.pdf (Visto última vez 01-03-2021).

LOMASKY, L., 1987. Persons, rights and the moral community, page 31-34. Oxford University Press.

MARX, K., 2004. El capital, tomo 1, pág. 99-104.

OECD 2019. Under pressure: the squeezed middle class, OECD publishing, Paris. . https://www.oecd.org/els/soc/OECDmiddle-class-2019-main-findings.pdf (Pag. 18).

OECD 2021. The organization for economic cooperation and development. https://knoema.com/IMFWEO2020Oct/imfworld-economic-outlook-weo-database-october-2020 (Visto última vez 10-03-2021).

PIKETTY, T., 2014. El capital en el siglo XXI, pág. 448. Harvard University Press.

SPI 2018. The social progress imperative. https://knoema.com/ SPI2020/social-progress-index (Visto última vez 01-03-2021). TAXATION AND CUSTOMS UNION, 2020. Taxation Trends in the European Union. 2020 Edition. https://ec.europa. eu/taxation customs/sites/default/files/taxation trends report 2020.pdf

Notas al final

${ }^{1}$ El trueque es el primer sistema mercantil conocido. Anterior a esa etapa, sólo existía la supervivencia, donde los productos sólo tenían valor de uso y no eran destinados al intercambio.

${ }^{2} \mathrm{El}$ dinero debe cumplir tres propiedades para ser considerado como tal: a) ser un medio de pago o de cambio aceptado y generalizado, b) representar una unidad de cuenta y c) consolidarse como una reserva o depósito de valor. 
${ }^{3}$ El valor de cada producto no está definido en base al coste de producción, sino en función a la ley de demanda y oferta

${ }^{4}$ Es común ver cómo se añaden "apellidos" a conceptos que tienen significado por sí solos, con la única intención de apropiarse de dichos significados y dotarlos de validez para que sirvan a sus propósitos: "derechos sociales", "violencia machista", "libertad sexual".

${ }^{5}$ Se basa en el planteamiento de cuán perjudicial es el ahorro en términos agregados cuando deriva de la suma del ahorro individual.

${ }^{6} \mathrm{El}$ socialismo teórico-marxista es un modelo de producción donde el aparato estatal está controlado por la dictadura del proletariado, quienes persiguen de forma continuada acumular la totalidad de los medios de producción, eliminando con ello el derecho de propiedad privada, lo que priva de un sistema económico que fije los precios transados en una sociedad.

7 La "burbuja" financiera que explotó en 2008-2009, tuvo su origen en un órgano de planificación central, como es el Banco Central. Por lo tanto, dicha crisis fue parte de la coacción sistemática del Estado en el ámbito financiero y bancario.

${ }^{8}$ Grecia evitó su salida del euro al haber sido rescatada por el Eurogrupo ( $1^{\circ}$ rescate de 110.000 millones en el primer semestre del 2010; $2^{\circ}$ rescate de 130.000 millones en el primer semestre del 2012 y; $3^{\circ}$ rescate de 85.000 millones en el segundo semestre del 2015). El rescate financiero y económico terminó en 2018.

9 El método apriorístico en la ciencia económica se explica mediante el axioma (a priori) de la acción humana.

10 Dentro del impuesto al trabajo, hay que considerar las cotizaciones sociales que paga, formalmente, el trabajador, y las que están a cargo de la empresa. En la medida en la que se quieran aplicar medidas similares a los países nórdicos, las cotizaciones sociales por parte de empleador deberán reducirse y aumentarse el porcentaje de IRPF.

Fecha recepción: 28 de enero de 2021

Fecha aceptación: 26 de mayo de 2021

Fecha versión final: 04 de junio de 2021 